\title{
Antibacterial Properties of Ag nanoparticle-loaded Multilayers and Formation of Magnetically Directed Antibacterial Microparticles
}

\author{
Daeyeon Lee $e^{\dagger}$, Robert E. Cohen ${ }^{\dagger *}$ and Michael F. Rubner ${ }^{\text {t* }^{*}}$ \\ ${ }^{\dagger}$ Department of Chemical Engineering, ${ }^{*}$ Department of Materials Science and Engineering and the \\ Center for Materials Science and Engineering, Massachusetts Institute of Technology, 77 \\ Massachusetts Avenue, Cambridge, Massachusetts 02139
}

Supporting Information

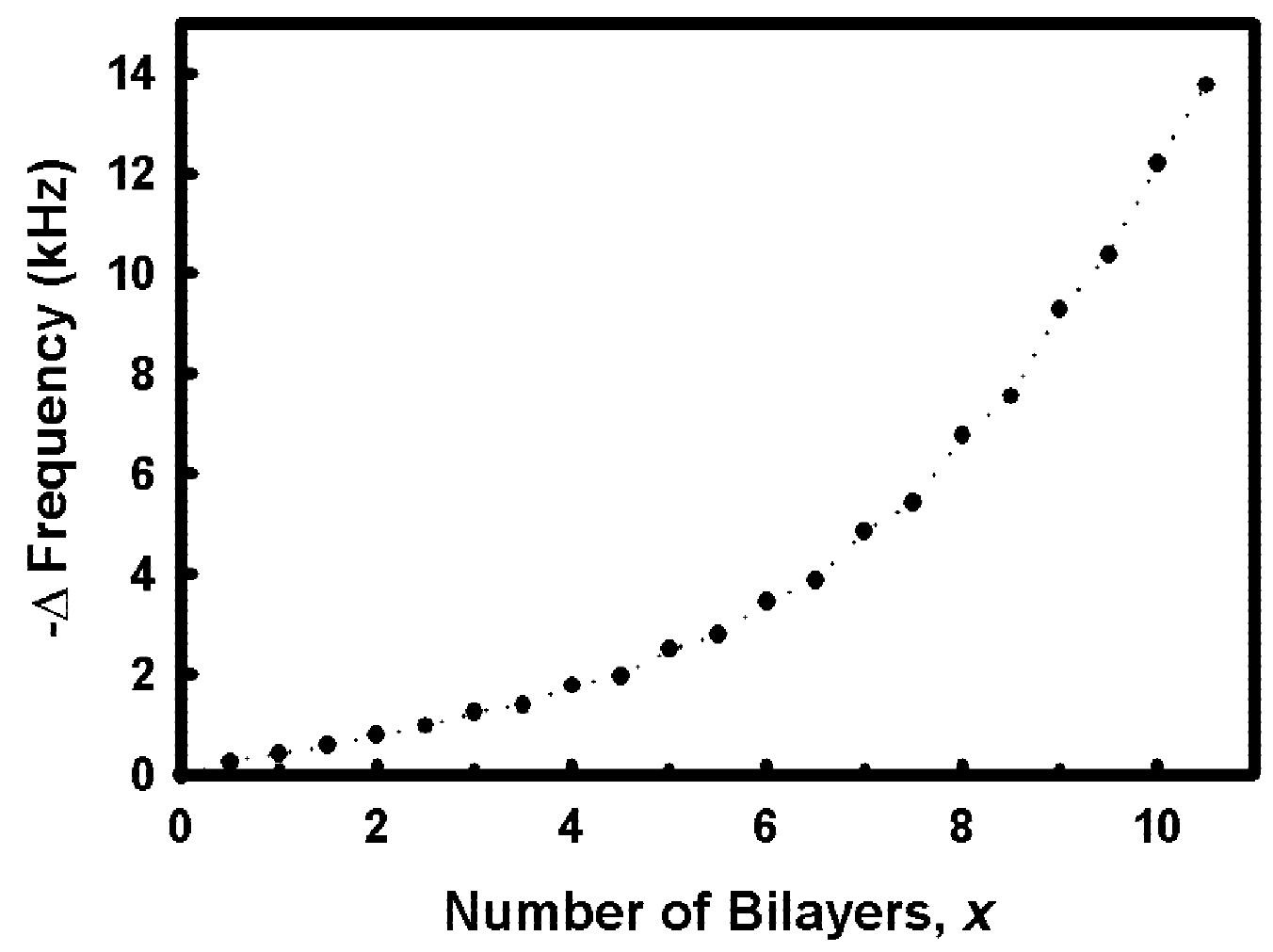

Figure S1. Frequency shift of the hydrogen-bonded multilayer comprised of poly(acrylic acid) (PAA) and polyacrylamide (PAAm) measured by quartz crystal microbalance (QCM) as a function of the number of bilayers $(x)$. 


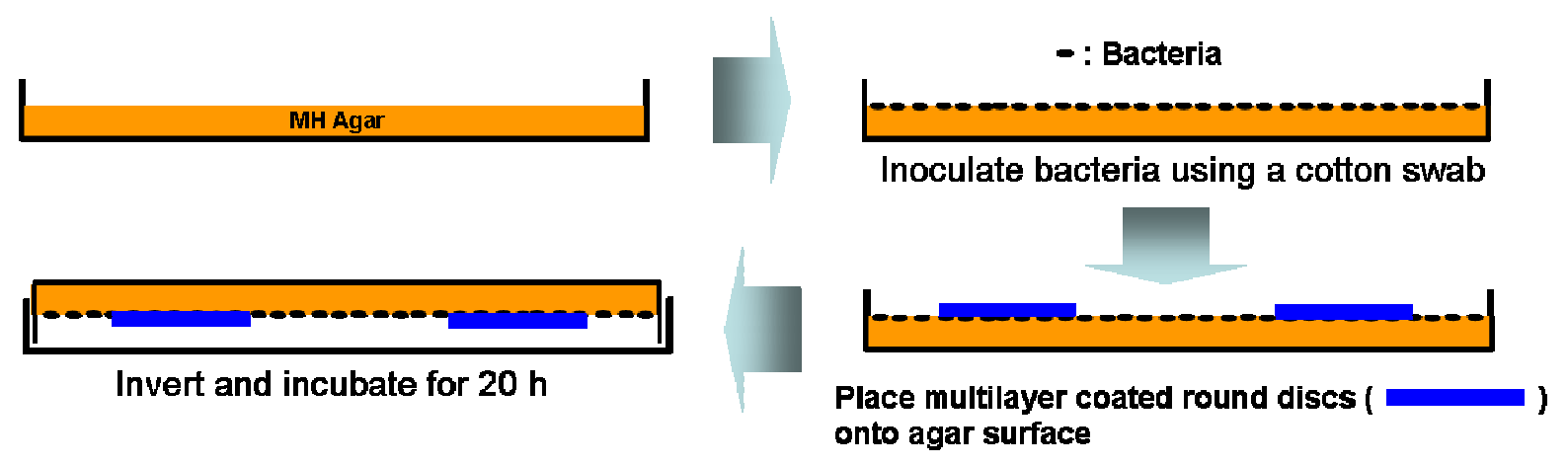

Figure S2. Kirby-Bauer method (disc diffusion test).
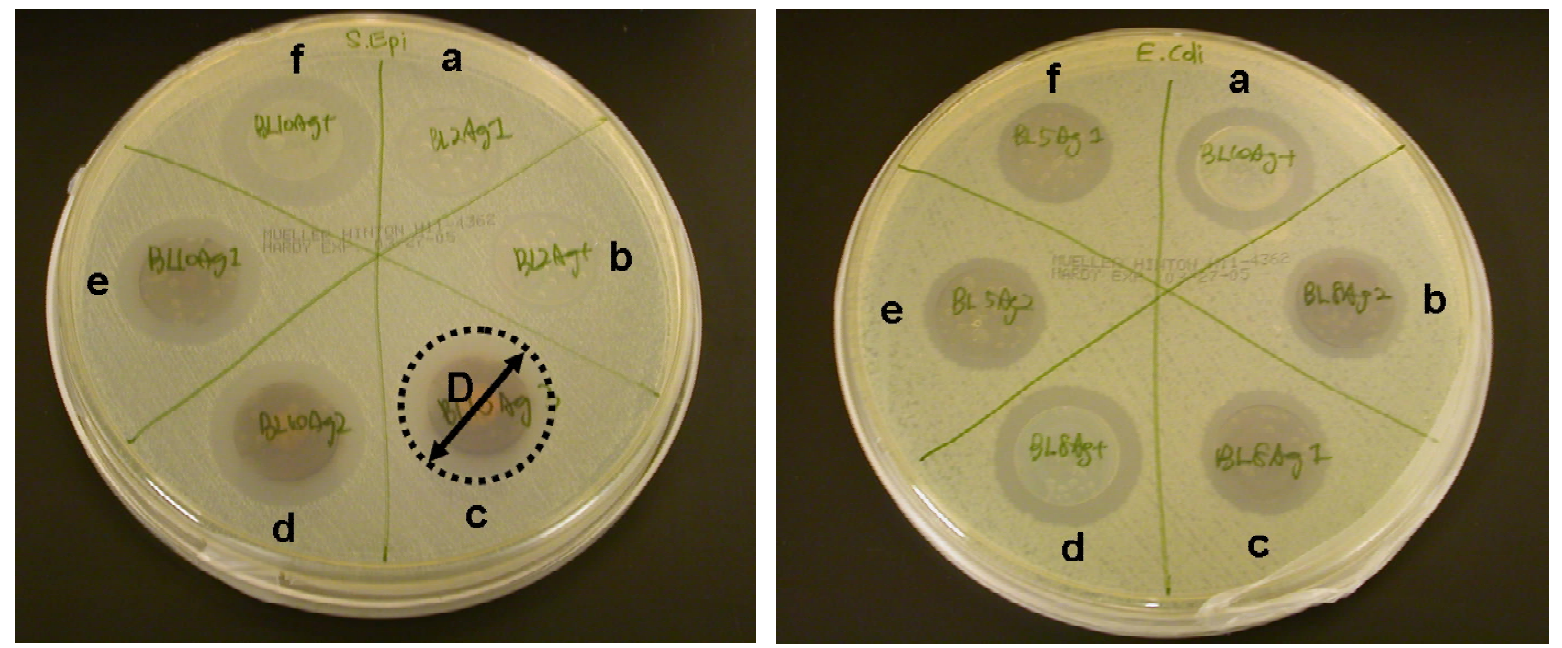

Figure S3. Images of MH agar plates after disc-diffusion tests were performed. S. Epidermidis (left) and E. Coli (right) were incubated for 20 hours. The zone of Inhibition is defined as [ZoI] = $\{$ D-diameter of disc $(22 \mathrm{~mm})\} / 2$. Each round disc is coated with $\mathrm{PAH}(\mathrm{PAA} / \mathrm{PAAm})_{x} \mathrm{Ag}_{n}$ films and placed on S. Epidermidis inoculated Mueller-Hinton (MH) agar (a) a: $x=2, n=1$, b: $x=2, n=+$, c: $x=10, n=3$, d: $x=10, n=2$, e: $x=10, n=1$, and f: $x=10, n=+$ and on E. Coli inoculated MH agar a: $x=10, n=+$, b: $x=8, n=2$, c: $x=8, n=1, \mathrm{~d}: x=8, n=+$ e: $x=5, n=2$, and f: $x=5, n=1$. 


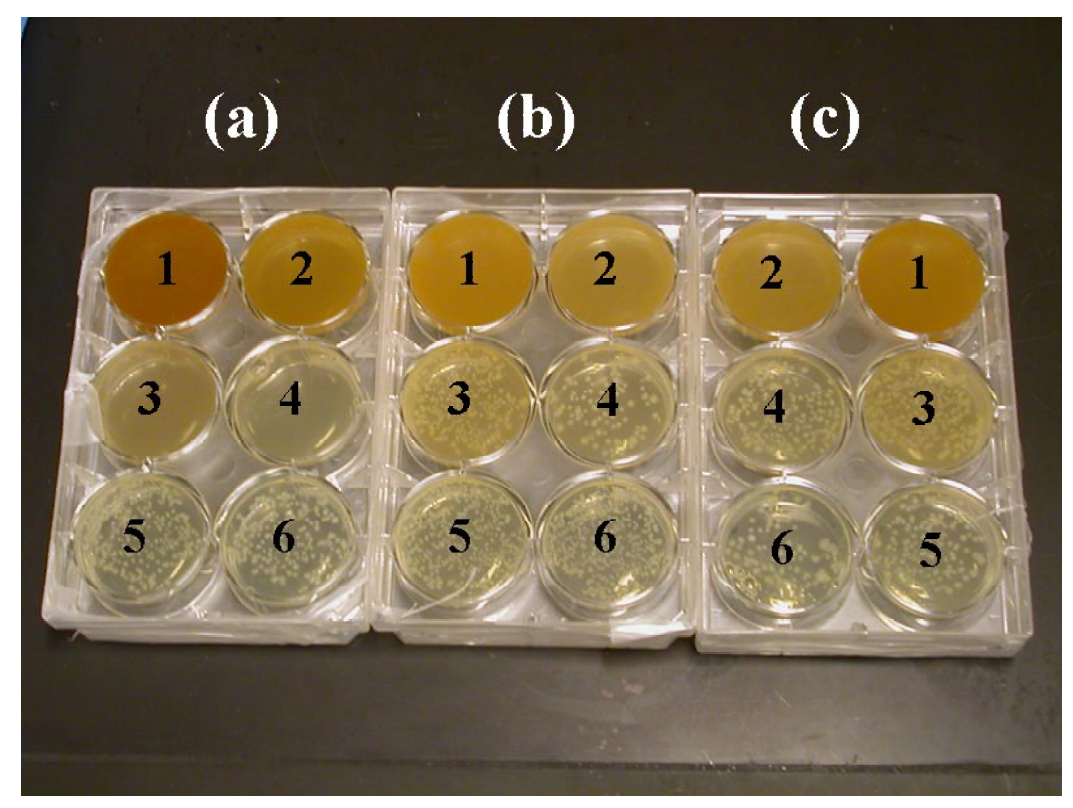

Figure S4. Image of plates used in the agar dilution technique performed after $20 \mathrm{hr}$ of E. Coli incubation. (a), (b) and (c) agar mixtures are made from a mixture of PAH(PAA/PAAm) ${ }_{4} \mathrm{Ag}_{3}$, $\mathrm{PAH}(\mathrm{PAA} / \mathrm{PAAm})_{4} \mathrm{Ag}_{+}$, and PAH(PAA/PAAm) ${ }_{4} \mathrm{Ag}_{1}$ suspension and $\mathrm{LB}$ agar. In the each 6-well plate, the concentration of magnetic particles with Ag nanocomposite coatings decreases from 800 ppm (well \#1) to 25 ppm (well \#6) by half dilutions. 